\title{
Tradition exégétique : âges, styles et formes d'une réception par le commentaire
}

\author{
Dominique Poirel \\ Institut de recherche et d' histoire des textes, Paris
}

Lorsque en moins de cinq ans surgissent des textes inconnus par dizaines, comme l' équipe animée par Dragos Calma nous en offre l'heureuse surprise, les conséquences scientifiques ne peuvent être qu'immenses, en nombre comme en étendue ${ }^{1}$. C'est d'une de ces conséquences que j' aimerais vous entretenir. Ce qui peu à peu paraît sous nos yeux, au fil des éditions critiques, c' est bien plus qu' une soixantaine d'ouvrages inédits; c' est un filon textuel massif et cohérent, qu' on croyait naguère se cantonner à l' Europe occidentale du XIII ${ }^{\mathrm{e}}$ siècle, mais dont on sait aujourd'hui qu'il se déploie des îles britanniques à la Pologne et à la Bohême, et s' étend du franciscain Roger Bacon, dans les années 1240, au dominicain Giovanni Crisostomo Javelli, mort en 1538.

L' ampleur et la rapidité de cette résurgence en font un objet idéal pour étudier ce qu' est, en soi, une tradition exégétique ${ }^{2}$. Ayant eu l'occasion d'étudier auparavant d' autres catégories de commentaires - sur la Bible, sur Aristote, sur Porphyre, sur le pseudo-Denys l' Aréopagite, sur le Liber de causis - j' aimerais, à l'occasion de cette rafale de découvertes, me poser par comparaison quelques questions générales sur ce qu' est une tradition exégétique: comment peuton définir ses contours, distinguer ses phases, souligner ses particularités? Par la force des choses, mes questions trop vastes demeureront longtemps sans réponse: pour y répondre il faudrait disposer d'éditions critiques, en cours mais trop nombreuses pour paraître avant des dizaines d'années. Venant trop tôt, mon exposé sera plus théorique et programmatique que je ne l' aurais souhaité. Il offrira des généralités, peut-être même des banalités. J' estimerai cependant son but rempli s'il parvient à vous convaincre de l'importance d'étudier chaque commentaire en relation avec sa tradition exégétique tout entière.

1 Voir en dernier lieu Calma 2016.

2 Sur le «commentarisme» antique et médiéval, la bibliographie est abondante. Pour la diversité des catégories de texte et des angles d' approche, on se bornera à mentionner deux colloques: Goulet-Cazé 2000 et Moulin (à paraître). 
Mais d'abord, qu' est-ce qu'une «tradition exégétique»? Comme l' expression se répand depuis les années 1980 en des sens subtilement proches mais divers, je dois préciser auquel je pense ici. Par «tradition exégétique», les uns signifient un courant exégétique, une certaine manière de comprendre tel livre, tel passage ou même telle réalité; tel l'article d'Antoine Bastiaensen, «La perdrix animal méchant figure du diable: Augustin héritier d'une tradition exégétique» ${ }^{3}$. Par «tradition exégétique», d'autres entendent une méthode exégétique, c' est-à-dire une certaine manière d'expliquer les textes, comme lorsqu' on oppose la typologie antiochienne et l' allégorie alexandrine ${ }^{4}$. Pour ma part, j'appellerai «tradition exégétique» l'ensemble des commentaires d'une même œuvre: c'est en ce sens que Cristina D'Ancona étudiait «Syrianus dans la tradition exégétique de la Métaphysique d'Aristote ${ }^{5}$. Dans la première acception, une tradition exégétique se distingue d'une autre par un choix interprétatif; dans la seconde, par un choix méthodologique; dans la troisième (celle que je vous propose), par le choix de l'œuvre à exposer. Une tradition exégétique sera donc pour nous un corpus de commentaires portant sur la même œuvre. «Corpus», «commentaire» et «œuvre»: ces trois notions demandent chacune des éclaircissements; elles structureront mon exposé.

Tout d'abord, «corpus». Les œuvres examinées ne sont pas indépendantes ou juxtaposées, mais forment une grande famille. Elles sont liées entre elles par des relations d'intertextualité, qu'il importe de démêler, car ces relations façonnent la tradition exégétique, lui conférant une structure d'ordre au sens mathématique. Or les relations entre commentaires peuvent être de deux sortes. Quand des œuvres émanent de maîtres contemporains, amis ou rivaux, qui se connaissent, se rencontrent, s'écoutent, se tiennent au courant de leurs enseignements et réagissent aux thèses l'un de l'autre, elles portent des traces d' influences croisées. Le plus souvent, toutefois, les relations d' œuvre à œuvre sont orientées dans le temps et unilatérales. L'une précède l' autre et l'influence, sans que la réciproque soit vraie. C'est par excellence le cas d'ouvrages que sépare un intervalle de temps et qui se sont transmis par écrit. Dans le pre-

3 Voir Bastiaensen 1990.

4 Voir O'Keefe 2014.

5 Voir D'Ancona 200o; voir aussi Cheng 1985; voir aussi, avec une expression analogue: Thijssen, Braakhuis 1999; Calma, 2016a, p. 11-52. 
mier modèle, les relations sont «horizontales» ou simultanées; dans le second, elles sont «verticales» ou successives.

Le temps est donc une dimension essentielle pour étudier les traditions exégétiques: il permet à l'observateur de les ordonner, tel un stemma textuum, en posant, tout en haut, l'œuvre à commenter, puis, de haut en bas, tous les commentaires successifs, étagés les uns sous les autres d'après la date de leur composition. Chaque commentaire est susceptible d'être influencé par un ou plusieurs de ceux qui le précèdent, mais entre eux les relations sont rarement uniformes et linéaires: certains rencontrent plus de succès que d'autres, ils forment comme des «nœuds» dans la tradition exégétique ${ }^{6}$. Au Moyen Âge, les traditions exégétiques se développent souvent par accumulation: chaque commentateur n' est qu'un maillon dans l'histoire d'un texte et de sa réception, mais cette histoire, considérée globalement, tend vers la prise en compte progressive du plus grand nombre d'interprétations accessibles, pour les additionner, les trier, les critiquer, les infléchir, les adapter, les enrichir et, chaque fois que possible, les concilier?

La question du temps se pose non seulement pour chaque commentaire, pris séparément - sa chronologie absolue - ou pris en relation avec tel ou tel autre texte - sa chronologie relative -; elle se pose aussi pour la tradition exégétique prise comme un tout. Chaque tradition exégétique est comme un cycle de Kondratiev: elle a ses phases, ascendante et descendante, et au milieu une phase intermédiaire. Je distinguerai donc trois moments dans le déroulement d'une tradition exégétique: l'âge des initiateurs, l'âge des continuateurs, l'âge des conservateurs.

\section{1 Âge des initiateurs}

L'âge des initiateurs est celui où se met en place la tradition exégétique. C'est une phase essentielle, décisive pour la suite. Une fois la tradition exégétique constituée, commenter le même livre deviendra souvent l' exercice obligé d'un parcours scolaire ou universitaire. Mais chez les premiers commentateurs, exposer tel texte plutôt qu'un autre obéit plus que jamais à des raisons personnelles. Ces raisons peuvent être conscientes, lorsqu'il existe une affinité profonde entre la pensée d'une œuvre et celle de son premier exégète, tels le corpus dionysien et l'Érigène. Elles peuvent être accidentelles, lorsqu' un exé-

6 Voir ci-dessous, dans le le stemma textuum du premier appendice, la position de Jean Scot et Hugues de Saint-Victor à l'intérieur de la tradition exégétique du pseudo-Denys latin des origines au début du XIII ${ }^{\mathrm{e}}$ siècle.

7 Ainsi, dans l' interprétation de la Bible, la Postille d'Hugues de Saint-Cher s' efforce de réunir et condenser la totalité des exégèses précédentes, voir Bataillon, Dahan, Gy 2004. 
gète se méprend sur la teneur doctrinale de l'œuvre qu'il expose. Nous pensons trop souvent que l' exégète ne se met au travail qu' après avoir saisi pleinement le sens de l' œuvre: son commentaire ne serait alors que la mise par écrit d'une interprétation acquise au préalable. En réalité, si commenter, c'est lire de la manière la plus attentive et la plus active qui soit, l'élucidation plénière de l'œuvre sera le résultat, bien plus que le point de départ, du labeur exégétique. Ce n' est qu' une fois son travail achevé que l' exégète comprend le mieux les raisons qu'il avait - ou non - de l' entreprendre. Même s'il avait déjà lu l'œuvre dans son entier, le premier exégète la redécouvre phrase après phrase, à mesure qu' il l' explique. Entre le début et la fin, sa perception de l'œuvre mûrit et se transforme peu à peu, à la lumière de tout ce qu'il a déjà commenté. Il y a de ce fait une part non négligeable de hasard et de pari dans le choix du premier exégète de commenter cette œuvre-ci plutôt que celle-là.

$\mathrm{Au}$ fond, les premiers exégètes sont comme des acrobates sans filet: ils doivent affronter seuls les difficultés du texte, sans pouvoir se rattraper à quoi que ce soit en cas d' erreur de transcription, de traduction ou d'interprétation. Dans son commentaire au De memoria et reminiscentia, Adam de Buckfield s'évertue à rendre intelligible un texte déformé par l'insertion de termes saugrenus: des noms propres que le traducteur a pris pour des noms communs et dont l'un a subi de surcroît une erreur de copie $^{8}$. Comme Adam ne manque pas de finesse, il parvient à intégrer au thème de la mémoire des réalités aussi improbables qu'un «tondeur» (Coriscos) ou qu' un «miroir de la montagne tenu tout autour» (Antiphéron d'Oreos) $)^{9}$. Quand les erreurs sont criantes, on les corrige dès que possible: celles que j' ai dites disparaissent dès la translatio nova par Guillaume de Moerbeke ${ }^{10}$. Mais il n' en va pas de même avec des glissements plus subtils, induits par le décalage culturel entre l'auteur et son premier commentateur. Le risque existe alors que des mauvais plis soient pris,

Julie Brumberg-Chaumont et moi préparons l'édition de ce commentaire pour la collection Auctores Britannici Medii Aevi, d'après les trois manuscrits: Bologna, Biblioteca Universitaria, $1180(2344)$, f. $54^{\mathrm{r}}-56^{\mathrm{v}}$; London, Wellcome Historical Medical Library, 3, f. $141^{\mathrm{va}}-144^{\mathrm{rb}}$; Vaticano, Biblioteca Apostolica Vaticana, Vat. lat. 5988 , f. $26^{\mathrm{rb}}-29^{\mathrm{va}}$.

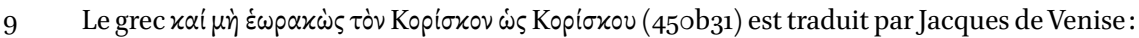
et non est considerans tonsorem ut tonsoris ymaginem, puis commenté par Adam: et ita considerat anima ipsam rem extra animam sicut 'tonsorem,' quando memoratur secundum 'ymaginem tonsoris' existentem in animam, in quantum tamen ejus ymago est. De même le


culo, mais on présume que l' abréviation de contra fut lue à tort circa puis circum, ce qui donne chez Adam: 'Ut accidit in circumferenti, speculo', id est retento; vel aliter 'in speculo de monte que circa ipsum fiunt', quasi dicat: 'ferente' ad visum que 'circa' ipsum sunt.

10 Respectivement: et qui non videt Coriscum ut Corisci ymaginem et ut accidit Antiferonti Oreite. 
qu' il sera très difficile ensuite de corriger ${ }^{11}$. Suivant une tendance cumulative qui affecte les traditions exégétiques, les explications erronées sont très résistantes, non moins au Moyen Age qu'à notre époque. En cas de divergence, les exégètes préfèrent rassembler toutes les thèses émises, avant de les discuter et si possible de les harmoniser: ce parti conservateur et conciliateur permet aux erreurs initiales de se perpétuer longtemps après qu' elles ont été réfutées, telle la paternité aristotélicienne du Liber de causis.

À l'âge des initiateurs, les exégètes produisent plus volontiers des gloses ou commentaires continus, à teneur plus grammaticale et moins doctrinale que par la suite. L' œuvre étant inconnue, il importe de l'étudier dans son entier et de «mâcher» une à une toutes ses difficultés de surface: obscurités lexicales et syntaxiques, ponctuation, renvois à d' autres textes ou réalités. C' est une fois achevé ce travail plus minutieux que les exégètes suivants pourront plus facilement saisir la pensée de l' œuvre et se concentrer sur les problèmes doctrinaux qu' elle soulève ${ }^{12}$.

Que font les premiers exégètes devant un passage énigmatique? Faute d'un commentaire antérieur, ils recourent naturellement à des textes analogues, ou jugés tels; ils bricolent avec ce qu' ils connaissent. De là une tendance du commentaire princeps à banaliser l'œuvre, à lui ôter tout ce qu' elle présente de neuf, de saillant, d' exotique. Ce n' est que peu à peu que les commentaires suivants rendront de plus en plus fidèlement les idées de l'auteur. Ainsi, dans le second commentaire latin à la Hiérarchie céleste, Hugues de Saint-Victor augustinise la pensée du pseudo-Denys ${ }^{13}$. Dans le premier commentaire aux Noms divins, Guillaume de Lucques l'interprète à travers les théories de son maître Gilbert de la Porrée ${ }^{14}$. Ce n' est qu'un siècle plus tard que Thomas Gallus, par son triple exégèse du corpus dionysien - glose, paraphrase et grand commentaire - parvient à rendre dans toute son étrangeté la pensée apophatique du pseudo-Aréopagite; encore n'est-ce pas sans conserver et même amplifier une ligne d'interprétation affective et psychologisante, présente en germe chez Hugues de Saint-Victor, et qui devait jouer ensuite un rôle majeur dans la lecture franciscaine et surtout chartreuse des écrits dionysiens ${ }^{15}$. Bref, le commentaire premier est comme la cause première: il agit plus vigoureusement et plus largement que le commentaire second sur la tradition exégétique.

\footnotetext{
11 Sur ces glissements, voir Poirel 2013, p. 397-404.

12 Voir notamment Weijers, Holtz 1997; Weijers 2011; Ead. 1996.

13 Voir Roques 1962.

14 Voir Gastaldelli 1983.

15 Voir Nemeth 2010, en part. p. 335-354; Rorem 2009; Poirel 2013.
} 


\section{2 Âge des continuateurs}

Après l'âge des initiateurs, vient l'âge des continuateurs. Les premiers ont défriché l' œuvre, la rendant accessible aux autres; ils ont aussi convaincu les générations suivantes que l' œuvre avait de la valeur et méritait d' être commentée à nouveau, peut-être même d'être inscrite dans les programmes scolaires ou universitaires: c' est alors le succès garanti pour un certain nombre de générations. Ce qui était à l' âge précédent une initiative isolée, individuelle, devient une pratique commune et institutionnelle.

Plus les commentaires se multiplient, moins il leur est possible d'être originaux: inévitablement ils se répètent, se citent, se compilent, se remploient les uns les autres, selon des relations intertextuelles de plus en plus complexes ${ }^{16}$. Des «vulgates» exégétiques se mettent en place, ainsi que des phénomènes d'écoles exégétiques: avec le temps on distingue, dans l'interprétation de l' œuvre, ce qui fait l'objet d' un consensus à peu près général (généralement ce qui ne pose pas grand problème doctrinal); et ce qui suscite au contraire des querelles et des débats entre exégètes. Prendre telle ou telle option sur l'interprétation de passages sensibles devient alors une marque d'affiliation, un signe d' allégeance à telle ou telle école. Bien sûr, les options adverses seront connues, formulées, discutées; mais pour être à la fin rejetées ou réinterprétées, anesthésiées, mithridatisées. Ces divergences exégétiques sont souvent un excellent marqueur pour distinguer les courants intellectuels qui structurent le paysage académique d' une époque ${ }^{17}$.

Les commentaires du second âge prennent souvent une forme discontinue: délaissant l' élucidation besogneuse du mot à mot, puisque d'autres l' ont déjà réalisée, ils peuvent se concentrer sur les passages ou les questions les plus cruciaux: typiques sont alors les commentaires sous forme de quaestiones ${ }^{18}$. On voit aussi, à l' inverse, se former une «glose ordinaire», c'est-à-dire une édition savante de l'œuvre, présentant son texte, tracé au centre de la page dans

16 Sur la notion de «plagiat», c' est-à-dire en réalité de «remploi », voir Brînzei 2011.

17 Voir Dahan 1999a, p. 113-114, qui tente au passage de définir les traits propres d'une « exégèse franciscaine, non seulement par une thématique spécifique (qu'il conviendrait de développer) et par le rôle conféré à la personnalité de saint François d'Assise, mais aussi par quelques caractères notables, comme l' assimilation des techniques contemporaines à une démarche spirituelle propre (je crois l' avoir montré à propos d'Antoine de Padoue) et, surtout, une attention à la lettre du texte, qu' il s' agisse de sa qualité matérielle ou du sens (et ainsi l'on n' est pas surpris de trouver au XIV siècle un prolongement de cette exégèse avec la Postilla litteralis d'un autre exégète franciscain majeur, Nicolas de Lyre).»

18 Sur les formes et méthodes de l'exégèse biblique, voir Dahan 1999b, en part. p. 121159 . 
une écriture de grand module, tandis que les interlignes et surtout les marges abritent une collection plus ou moins stable de notes exégétiques, extraites de commentaires antérieurs et censées en fournir la quinte essence. Cette annotation continue, fragmentaire et périphérique se prête à une double lecture: simple et linéaire, ou transversale et hypertextuelle grâce au va-et-vient entre l'œuvre et sa glose. De telles «gloses ordinaires» sont bien connues pour la Bible: elles ont été inaugurés à Laon au tournant du XII ${ }^{\mathrm{e}}$ siècle ${ }^{19}$. Il en existe de comparables pour les écrits du pseudo-Denys: dès la fin du IX ${ }^{\mathrm{e}}$ siècle Anastase le Bibliothécaire a inauguré une édition annotée de la version érigénienne, qui se développe au fil du temps et se stabilise en partie dans le courant du XIII ${ }^{\mathrm{e}}$ siècle, devenant ce que Hyacinthe Dondaine a nommé le «Corpus dionysien de l' université de Paris ${ }^{20}$. Des tentatives analogues existent aussi pour le corpus aristotélicien, par exemple dans la «Glose d' Oxford» élaborée au XIII ${ }^{\mathrm{e}}$ siècle, peut-être dans l' entourage d'Adam de Buckfield ${ }^{21}$. Enfin l' exposé d'Olga Weijers dans ce colloque nous instruira peut-être sur l' existence d'une «glose ordinaire » du Liber de causis ${ }^{22}$.

\section{3 Âge des conservateurs}

Après l'âge des initiateurs et des continuateurs, vient celui des conservateurs, plus difficile à décrire. Vient en effet le moment où peu à peu l'importance du texte s'érode dans la culture savante. Pourquoi ? Plusieurs explications peuvent concourir. Ce peut être d'abord parce que l' interprétation initiale, dont je disais la tendance banalisante, a cédé le pas à une interprétation plus fidèle, plus authentique, et que celle-ci se trouve révéler le caractère décevant, «hérétique » ou inassimilable de l' œuvre au regard de la pensée dominante ${ }^{23}$. Encore que cette situation peut à l'inverse stimuler l'activité exégétique, soit pour mettre en lumière le désaccord ainsi apparu, soit pour le nier à l'aide d' explications accommodatrices ou allégorisantes ${ }^{24}$.

19 Voir par exemple l'étude pionnière de Smalley 1961; Smith 2009. Voir aussi le site glossae .net.

$20 \quad$ Voir Dondaine 1953.

21 Voir Galle 2008.

22 Voir dans ce volume la contribution d' Olga Weijers: «Les gloses sur le Liber de causis dans les manuscrits parisiens».

23 Après la mort d'Averroès, on observe dans le monde musulman un certain déclin de la tradition aristotélicienne, jugée infidèle à l' islam sunnite.

24 C' est par exemple le cas de l' exégèse chartraine de textes platoniciens ou néoplatoniciens, grâce à la théorie de l' integumentum ou de l' involucrum, voir Chenu 1955; Jeauneau 1973; Stock 1972. 
Ce peut être à cause de la découverte ou production d'autres textes, qui déclassent l' œuvre et la rendent obsolète ${ }^{25}$. À elle seule toutefois cette cause suffit rarement: le Moyen Âge et en particulier le Moyen Âge scolastique se montre plus soucieux d'accumuler indéfiniment les autorités et de valoriser leur ancienneté, plutôt que de faire le ménage parmi elles pour ne retenir que les plus récentes.

Ce peut être par lassitude. On peut imaginer qu' une tradition exégétique se soit montrée si efficace, soit si bien parvenue à expliquer les difficultés d'un texte, à trancher ses indécisions, bref à le rendre accessible, intelligible, clair, évident, donc banal pour l'ensemble des lecteurs, qu'il n'y ait plus besoin d'interposer entre ceux-ci et l' œuvre elle-même la médiation d'une exégèse: le recours direct aux originaux déclasse alors les commentaires ${ }^{26}$. Ou encore, l'un ou quelques-uns des commentaires anciens remplissent si bien leur rôle qu' ils escortent officiellement l' œuvre dans les manuscrits et deviennent classiques au même titre qu' elle $^{27}$; on en vient à ne plus distinguer l'œuvre de son compagnon ordinaire et, à proprement parler, on n'écrit plus de commentaires de l'œuvre, mais des sur-commentaires, des explications au carré de l'œuvre via son commentaire ordinaire ${ }^{28}$. Dans ces deux derniers cas, la tradition exégétique (directe) s' éteint, victime de son succès.

Ce peut être enfin, et le plus souvent, à cause de mutations culturelles importantes, qui conduisent les intellectuels, ou une partie d'entre eux, à rejeter des autorités tenues pour traditionnelles jusqu' alors, au profit d'autres autorités, plus neuves, ou au contraire plus anciennes, comme ce fut le cas à la

25 C'est le cas des sources péripatéticiennes, qui ont dans une certaine mesure tari la tradition exégétique de sources néoplatoniciennes comme Macrobe, Calcidius et Martianus Capella, courantes encore au XII ${ }^{\mathrm{e}}$ siècle.

26 Ce serait par exemple le cas du Liber de causis, s'il était bien composé de propositions et de leur commentaire, comme l'ont pensé de nombreux exégètes médiévaux.

27 Sans aller jusque-là, les exégètes carolingiens se montrent si soucieux d' expliquer la Bible selon les Pères que leurs commentaires successifs sont de véritables patchworks - souvent très proches les uns des autres - d' explications tirées des ouvrages patristiques. Ce qui fait dire à Silvia Cantelli: «Il tratto distintivo dell'esegesi carolingia fu quello di aver definito un ambito di esercizio interpretativo dove la tradizione svolge un ruolo determinante, tanto da sovrapporsi fino a sostituirsi completamente al testo sacro» (Cantelli 199o, p. 68).

28 Dans une certaine mesure, la Glossa ordinaria tendit ainsi à se substituer à la Bible elle-même au cours des $\mathrm{XII}^{\mathrm{e}}-\mathrm{XIV}^{\mathrm{e}}$ siècles, au point que les maîtres exposent l'une et l'autre ensemble. Cette pratique d'une «glose de glose» se matérialise dans les marges des manuscrits d'enseignement: au centre le texte biblique en lettres de gros modules; tout autour, la Glose ordinaire en lettres de modules plus petites; dans les marges, le surcommentaire peut-être oral d'un maître, noté par lui-même ou par un étudiant. Voir par exemple le manuscrit Paris, BnF, lat. 17232, dont on trouvera, p. 184, la reproduction du f. $75^{\mathrm{r}}$, dans Lobrichon 1990, p. 181-185. 
Renaissance. Certaines traditions exégétiques tout entières: celle d'Aristote, celle des Sentences de Pierre Lombard, celle du Liber de causis, ne sont pas pour autant rejetées, mais reléguées ${ }^{29}$. Il se produit dans le monde savant une scission culturelle entre, d'un côté, les cercles humanistes, tournés vers les textes antiques, classiques et patristiques; et, de l' autre, les milieux universitaires, qui perdurent mais changent ${ }^{30}$. Se met alors en place une «seconde scolastique ${ }^{31}$. Dans le cas de la Renaissance, cette scission culturelle conduit les cercles humanistes à rejeter non seulement certaines traditions exégétiques, mais encore certaines méthodes exégétiques liées à l'université, comme la glose, le commentaire syllogistique, le commentaire par quaestiones ${ }^{32}$. C' en est fini d'une certaine exégèse: le commentaire, et d'abord une certaine manière de commenter, a cessé d'être le paradigme dominant du labeur intellectuel.

Quoi qu'il en soit, l'extinction d'une tradition exégétique tout entière ne s'opère pas brutalement: il y a donc une période finale dans laquelle se livrent des combats d'arrière-garde. Dans cette tradition finissante, ceux qui choisissent, contre les tendances nouvelles, de commenter les autorités anciennes font figure de conservateurs. Néanmoins, puisqu' ils sont devenus minoritaires, leur décision fait, comme au début, l' objet d'un vrai choix individuel, d' autant plus signifiant pour l'historien. Conservateurs, mais dans des circonstances inédites, traditionnels à rebours de traditions naissantes, les voici contraints de mêler l'audace à la réaction, parfois même d'innover pour combler l'écart croissant entre leur époque nouvelle et leur projet périmé: ils sont modernement démodés ${ }^{33}$.

Peut-on interpréter de cette manière les derniers commentaires du Liber de causis, en particulier celui du dominicain bolonais Crisostomo Javelli, mort en 1538 , remarquable à la fois par le formalisme de son commentaire et sa

29 Voir par exemple, Bianchi, Randi, 199o; Bianchi 2003; Bianchi 2011; Franck, Speer 2007.

30 Voir Blaise Dufal, «Scolastique et humanisme: paradigmes concurrents et contemporanéité des épistémès», dans Foucault et la Renaissance, colloque organisé à l'Université Toulouse II - Le Mirail les 13-16 mars 2012. En attendant la parution des actes, la communication est accessible en vidéo à l' adresse: $h$ ttps://www.canal-u.tv/video/universite_toulouse _ii_le_mirail/scolastique_et_humanisme_paradigmes_concurrents_et_contemporaneite _des_epistemes_blaise_dufal.11013.

31 Voir les travaux de Jacob Schmutz, en particulier son «Bulletin de scolastique moderne», dans Revue thomiste, t. 100, 2001, p. 27-341, ainsi que le site internet qu'il a créé: Scholasticon, Ressources en lignes pour l'étude de la scolastique moderne (1500-1800). On y trouve notamment un index alphabétique des scolastiques de l'époque moderne: http:// scholasticon.ish-lyon.cnrs.fr/.

32 Voir Boulègue 2014.

33 Par exemple sur John Mair, maître à Paris au XVI ${ }^{\mathrm{e}}$ siècle, voir Biard 1986; ou encore Slotemaker, Witt 2015 . 
double préoccupation, synthétique et critique? Le titre même de son ouvrage le présente comme une epithoma et des examina ${ }^{34}$. Il se propose moins de commenter que de résumer et de juger. En effet, dit-il dans un bref prologue, le Liber de causis contient de nombreuses ambiguïtés, en sorte qu' il est difficile de savoir en quel sens plusieurs d' entre elles doivent être acceptées ou réprouvées. Aussi notre exégète divise-t-il chaque proposition en thèses ou «conclusions », qui sont ensuite examinées selon une «quadruple voie»: d'abord il résume la thèse de l'auteur, ensuite il la confronte avec la pensée de Proclus, puis d'Aristote, puis du «Théologien», qui est moins un théologien précis que le porte-parole anonyme de la théologie catholique, thomiste de préférence. Pour finir, il conclut en déclarant dans quelle mesure la thèse examinée peut être acceptée pour vraie ${ }^{35}$.

Avec Javelli, nous sommes typiquement au terme d'une tradition exégétique: son but n' est plus d'expliquer une auctoritas pour elle-même, dans la conviction qu'elle alimentera la recherche du vrai, mais de résumer et d'évaluer un texte par rapport à des doctrines néoplatonicienne, aristotélicienne et chrétienne déjà constituées. En d'autres termes, la lettre du texte a cessé d'importer: on se contente d' en résumer les idées essentielles. L'œuvre a perdu son statut d'auctoritas: elle n'est plus qu'une source respectable, mais partiellement suspecte, réclamant un tri. L' œuvre ne soutient plus l'étude d'une discipline, la théologie; elle est au contraire jugée selon les conclusions de cette discipline théologique. Il y a donc du procès dans le commentaire de Javelli, qui

34 Le titre complet, dans l'édition de Venise 1531, est: Epithoma Reverendi Patris Fratris Crisostomi Javelli Canapitii: Ordinis Predicatorum: Sacre Theologie Professoris Celeberrimi: super Propositiones Libri de Causis: et unicuique Propositioni apposito Commento: ac etiam Examina earumdem. Il se lit au recto d' un feuillet «1», après divers commentaires de Javelli, dont chacun a sa foliotation propre: sur la Physique ( $48 \mathrm{ff}$.), le De caelo et mundi (32 ff.), le De generatione et corruptione ( $28 \mathrm{ff}$.), les Meteora (40 ff.) et les Parva naturalia d'Aristote ( $44 \mathrm{ff}$.). Le commentaire au Liber de causis ( $42 \mathrm{ff}$.) occupe ce qui serait, dans une foliotation continue, les feuillets 193 à 234 du volume dans son entier; il est suivi d'un commentaire au De anima (32 ff.), d'un Tractatus de transcendentibus previus Metaphysice (14ff.) et d' un commentaire à la Metaphysica d'Aristote (112 ff.). Le volume entier s'intitule: Preclarissimum (!) Epitoma super totam naturalem Philosophiam et Metaphisicam Aristotelis:necnon subtilissima enucleatio propositionum omnium in Librum de causis: nuperrime ad lucem editita (!): auctore [...] Chrysostomo Javello Canapitio. Impressum Venetiis: in aedibus Francisci Bindoni: ac Maphei Pasini sociorum: ad instantiam Joannis Marqualis, 1531. Die 19 Maii. Sur Crisostomo Javelli, voir Tavuzzi 199 o and 1991; Tavuzzi 2007; von Wille 2004. Voir aussi le site Scholasticon à l' adresse indiquée en note 25, en part. http://scholasticon.ish-lyon .cnrs.fr/Database/Scholastiques_fr.php?ID=20.

35 On lira un échantillon de cette méthode dans notre édition partielle en annexe, p. 3641. 
instruit le caractère douteux du Liber de causis et le condamne indirectement à une sorte d'oubli exégétique.

Voilà ce qu' on peut dire concernant les phases d'une tradition exégétique; mais il faut prendre garde que la situation peut être plus complexe. En effet, j' ai tenté de montrer que la forme et la méthode d'un commentaire donné varient selon la place de ce commentaire dans sa tradition exégétique tout entière. Toutefois, elles peuvent être affectées aussi par l'époque où ce commentaire a été composé. Dès lors que les mêmes maîtres ont commenté des ouvrages aussi divers que la Bible, les classiques, Boèce, Macrobe, le pseudo-Denys, Pierre Lombard, Aristote et le Liber de causis, il est fatal que les pratiques d' une tradition exégétique plus ancienne affectent les pratiques d'une tradition exégétique plus récente. Bref, un certain synchronisme des méthodes tempère de chronologie absolue la chronologie relative, propre à chaque tradition exégétique, dont je viens de décrire les trois âges. Par exemple, on n' a pas attendu le Liber de causis pour mettre au point des procédures exégétiques par divisio textus, paraphrase, glose et quaestiones. De ce fait, les premiers commentaires latins du Liber de causis ressemblent largement aux derniers commentaires médiévaux d'Aristote, de Boèce ou de Porphyre. Il importe donc de faire le partage entre ce qui est spécifique à telle tradition exégétique, étant donné les singularités de l'œuvre commentée, et ce qui est commun à l'ensemble des traditions exégétiques contemporaines, qu' elles soient récentes ou anciennes. Tout n' est pas original, mais tout n' est pas transposable. Il y a donc deux temporalités différentes à prendre en compte et à concilier.

Pour conclure sur notre premier point, la tradition exégétique apparait avant tout comme un corpus d' œuvres exégétiques. Si originale soit-elle, chaque commentaire ne se comprend bien que rapporté à ce corpus, de même que la nature et le rôle d'un membre ne se comprennent bien que rapportés à l'organisme vivant tout entier. Cela signifie que pour bien comprendre chaque élément du corpus, il ne suffit pas de l'examiner en lui-même: il faut aussi prendre en compte sa place dans le corpus, c'est-à-dire sa position dans une chronologie relative et ses relations avec les autres commentaires, antérieurs, postérieurs ou contemporains. De ce fait nous sommes, pour interpréter les rares commentaires édités du Liber de causis, dans une situation inconfortable: une partie de leur signification historique et doctrinale demeurera cachée, tant que tous les autres commentaires ne seront pas accessibles... ce qui demande au bas mot quelques dizaines d' années. C' est la raison pour laquelle mon exposé ne peut être que prospectif. 
Le corpus dont on vient de parler est un corpus de «commentaires » : il convient à présent d' examiner ce deuxième mot de notre définition. À l'évidence, le mot de « commentaires» doit être pris ici en un sens large. Il peut s' agir en effet d'un commentaire continu, de gloses discontinues, de quaestiones suivant le fil du texte, d'un accessus introductif, d' une paraphrase explicative, d' une reformulation sous forme de syllogismes, ou de toute autre forme s'écartant de celles-ci ou les mélangeant librement, du moment qu' on désigne un texte se référant à un autre texte pour l' expliquer.

Un commentaire, au sens large, c'est donc un texte qui a son centre de gravité non en lui-même, mais dans un texte autre, antérieur et jugé majeur: ce qu'au Moyen Âge on nomme une auctoritas: ouvrage de référence faisant autorité dans un champ du savoir ${ }^{36}$. L'existence de commentaires suppose toute une organisation du savoir, dans laquelle il y a d'un côté un corpus d'auctoritates, de l' autre une communauté de lettrés, et entre ce corpus et cette communauté des relations symbiotiques. Les auctoritates tirent leur prestige de l'importance fondatrice que leur attribuent les lettrés; les lettrés tirent leur ascendant de la maîtrise dont ils font preuve dans la connaissance des auctoritates. Le commentaire est donc l'acte par lequel un lettré s'affirme comme lettré, et confirme une auctoritas comme auctoritas. La tradition exégétique est ce même acte, étendu d'un lettré individuel à sa communauté, aussi longtemps que celle-ci reconnaît à l' œuvre sa fonction d'auctoritas. On voit donc que l'étude, non seulement des commentaires, mais des traditions exégétiques prises comme un tout, est un excellent moyen d'entrer dans la culture savante d'une époque.

Mais qu' est-ce au juste que commenter? Si l'on en croit le Dictionnaire étymologique de la langue latine d'Ernout et Meillet, commentari est apparenté au substantif mens et aux verbes mentiri, reminisci ${ }^{37}$. Commenter, c'est à la fois penser, imaginer et se souvenir. Il s'agit en somme de réfléchir, de méditer, en l'occurrence sur un texte; d' être inventif, presque mensonger; et pour finir, de le remettre en mémoire et de le restituer. Le commentaire, comme l' art selon Cocteau, est un mensonge qui dit la vérité38 ${ }^{3}$ Il s' éloigne tactiquement du texte,

\footnotetext{
$36 \quad$ Voir Zimmermann 2001.

37 «Commentor, -āris, ātus sum, āri», dans Ernout, Meillet 2001, p. 397, $2^{\mathrm{e}}$ colonne.

38 «Je suis un mensonge qui dit toujours la vérité», dans Journal d'un Inconnu, Paris, 1953, p. 143, reprenant «Le matin je me penche... Je tombe de fatigue, de douleur, de sommeil... Comprenne qui pourra: je suis un mensonge qui dit toujours la vérité.», in Opéra, 1927, rééd. Paris, 1967 , p. 86.
} 
mais pour mieux y revenir. S'il dit autre chose, c'est pour faire mieux comprendre la même chose. Il interprète, actualise, maintient vivante une lettre qui sans lui deviendrait lettre morte.

Selon l'œuvre à commenter et les visées de son exégète, commenter peut consister à élucider des obscurités; à préciser une signification quand elle est douteuse, à l' infléchir quand elle semble incorrecte; à soutenir des affirmations par le raisonnement ou par la citation d' autres auctoritates; à discuter ces mêmes affirmations pour les concilier entre elles, ou pour les concilier avec d' autres affirmations d' autres œuvres, d' autres auctoritates. Dans tous les cas, commenter revient à un travail d' adaptation entre un texte et ses lecteurs, pour combler la distance, temporelle, géographique, linguistique ou culturelle qui sépare un texte d'ailleurs ou d' autrefois de ses lecteurs présents.

Commenter est donc une activité principale, dans l'histoire de la réception d'une œuvre, mais ce n'est pas la seule. Le travail mené pour recevoir une œuvre déborde de toute part l'acte exégétique, en amont et en aval. Avant de commenter l'œuvre, il y a un labeur préalable pour l'établir: sa traduction, quand l' œuvre a été composée dans une autre langue; sa correction et son édition, quand non seulement le sens général de l'œuvre importe, mais aussi sa lettre précise (ainsi de la Bible, des poètes, des philosophes), et que la lettre de cette œuvre a subi des variations telles que son unité se trouve en danger: l'œuvre cesse de faire référence quand il n' est plus possible de s'y référer avec certitude; et les lettrés cessent d'être reconnus comme tels quand ils ne sont pas sûrs de lire la même œuvre.

Une fois l'œuvre commentée et recommentée, sa réception ne s'achève pas là, mais se poursuit par d'autres moyens: par la citation, le remploi ou l'imitation dans des œuvres nouvelles, qui cette fois ont en elles leur propre centre de gravité; et au-delà, par une inspiration de plus en plus libre à mesure qu' elle est moins avouée. L' œuvre est définitivement reçue quand ceux qu' elle a marqués de son empreinte la reprennent à leur compte sans le dire, parce qu' ils la réutilisent sans le savoir ${ }^{39}$.

Dans l'histoire de la réception d' une ouvre, le labeur exégétique est donc un moment intermédiaire entre l'établissement du texte comme tel et sa disparition comme texte au profit d'une absorption, d' une digestion par la culture

39 Pour le corpus pseudo-aréopagitique, grâce à son lexique hautement reconnaissable, on peut pister des influences si superficielles ou détournées que l'origine dionysienne des notions employées pourrait être devenue inconsciente. De son vocabulaire de la transcendance divine, on finit par ne retenir qu'une sorte d'impact rhétorique, si bien qu'il peut être appliqué aux réalités les plus diverses. Voir Poirel 2001, repris dans Poirel 2013, p. 243-270. 
d' arrivée. C' est aussi un moment volontaire et conscient: on ne commente pas un texte par ignorance ou par distraction. C' est enfin un moment instrumental: le commentaire n'a pas sa fin en lui-même, mais il est ordonné, d' un côté, à l' œuvre qu'il commente, pour la rendre plus accessible; de l' autre, à la communauté des lecteurs présents, pour la rendre plus savante.

\section{3 \\ OEuvre}

La tradition exégétique est donc un corpus de commentaires, mais qui portent tous sur la même œuvre. On a vu qu' une œuvre, par le fait qu' elle soit commentée, jouit (au moins pour son exégète) du statut d'auctoritas, d'ouvrage fondamental et de référence. Combien plus quand la même œuvre donne lieu non pas à un commentaire unique, mais à une longue série de commentaires: c' est toute une communauté de lettrés qui lui reconnaît ce statut d'auctoritas. Le réseau de leurs relations personnelles, institutionnelles ou culturelles se reflète et se révèle à travers le réseau des relations intertextuelles entre leurs commentaires.

Mais de quoi parle-t-on quand on dit «la même œuvre»? Il peut s' agir d'un texte unique, comme dans le cas qui nous occupe, celui du Liber de causis. Mais la notion d'œuvre peut aussi se réfracter, de diverses manières. Tout d'abord, une même œuvre peut avoir fait l'objet de remaniements par l' auteur, ou par un autre après lui. Elle peut aussi être parvenue dans le monde latin à travers diverses traductions, indépendantes ou se contentant de remanier la précédente ${ }^{40}$. Dira-t-on alors que chaque version du texte a donné lieu à une tradition exégétique distincte, ou bien que toutes ensemble elles forment une tradition exégétique plus large, plus complexe, qui se structure et se ramifie selon les versions commentées? Les deux sont envisageables, mais il sera sûrement plus riche de privilégier l' acception large, car un exégète peut panacher plusieurs versions du texte qu'il commente. Il peut aussi choisir une version principale, mais connaître les autres et s' en servir pour proposer des interprétations alternatives dès qu' il butte sur les obscurités ou difficultés de la première: $c^{\prime}$ est ce qu' ont fait couramment les exégètes du pseudo-Denys aux XIII ${ }^{\mathrm{e}}$-XIV ${ }^{\mathrm{e}}$ siècle ${ }^{41}$.

40 Voir Contamine 1989; voir aussi les volumes de la collection The Medieval Translator, Turnhout, $1996 \rightarrow$ (16 volumes parus).

41 Cela leur était facilité par la possibilité de recourir à diverses versions latines et à leurs commentaires, grâce au «Corpus dionysien de l'université de Paris» dont l'histoire a été reconstituée par Dondaine 1953. 
On vient de parler de traductions: il est fréquent que la même œuvre suscite diverses traditions de commentaires dans diverses langues ${ }^{42}$. Là aussi parlera-t-on de plusieurs traditions exégétiques, une pour chaque langue, ou bien d'une seule qui les réunit? À nouveau, les deux sont possibles, mais dès lors que certains commentaires aussi ont été traduits d'une langue à l' autre comme ceux d'Averroès sur les ouvrages d'Aristote - il paraît utile de privilégier une approche globale. En ce sens, il y a, par exemple, une tradition exégétique du De anima d'Aristote, tout autour de la mer Méditerranée, et cette tradition exégétique unique se démultiplie en sous-traditions, qui ne sont pas étanchément séparées, mais communiquent entre elles, de façon parfois surprenante, comme dans le cas du grand commentaire d'Averroès que ressuscitent Marc Geoffroy et Colette Sirat à partir du manuscrit de Modène, dans lequel ce grand commentaire, auparavant connu par sa version latine seulement, surgit à travers des gloses marginales en langue arabe et en caractères hébraïques ${ }^{43}$. Mieux que tout autre, ce cas extrême montre à quel point on ne peut, sans risque d' appauvrissement scientifique, cantonner à une langue la notion de «tradition exégétique».

La notion d'œuvre se réfracte encore d' une autre manière: à côté de textes clairement distincts, comme le Liber de causis, d'autres se présentent comme des textes gigognes, en sorte qu'on peut se demander si la notion d'œuvre s'applique à l'ensemble ou à chacun de ses éléments. C' est par exemple le cas des douze petits prophètes de l'Ancien Testament, des treize - ou quatorze lettres de saint Paul dans le Nouveau Testament, des dix ou onze lettres du pseudo-Denys l'Aréopagite, des cinq Opuscula theologica de Boèce, des sept à neuf Parva naturalia d'Aristote, et ainsi de suite. Dans l'optique qui est la nôtre, le parti le meilleur sera probablement de considérer comme formant une même œuvre tout recueil d'opuscules régulièrement commentés à la suite.

Mais alors, où s'arrêter? Car au Moyen Âge les textes ont une irrépressible tendance à s' assembler en des corpus arborescents, si bien que la notion d'œuvre est susceptible de se démultiplier presque indéfiniment en textes de plus en plus brefs, ou au contraire de se fondre dans des œuvres ou corpus de plus en plus larges. Pour reprendre un des recueils d' opuscules qu' on vient de mentionner: le livre d'Osée entre dans le recueil des petits prophètes; celuici entre, avec les grands prophètes Isaïe, Jérémie, Ézéchiel et Daniel, dans le groupement plus ample des textes prophétiques; lequel entre à son tour avec

\footnotetext{
42 Un inventaire des traductions (et commentaires) d'auteurs anciens, grecs et latins jusqu' en 1600 a été lancé en 1946 et soutenu ensuite par l'Union académique internationale: Kristeller $1960 \rightarrow$.

43 Sirat, Geoffroy 2005 .
} 
d' autres livrets bibliques, dont la liste varie d'un canon à l' autre, dans le corpus des «livres prophétiques»; qui forme, après le Pentateuque et avant les écrits des hagiographes, l'une des trois parties de l'Ancien Testament ${ }^{44}$.

La Bible n'est pas un cas isolé: l'œuvre d'Aristote se présente elle aussi comme un tel corpus savamment hiérarchisé, selon un classement dont l' origine remonterait à Andronicos de Rhodes ${ }^{45}$. Par exemple le De memoria et reminiscentia, qui se décompose d' ailleurs en deux parties ou traités, est rangé d'ordinaire dans les parva naturalia d'Aristote; cette collection d'opuscules physiques est à son tour rangée, avec des traités plus grands, notamment une Physica au féminin singulier, parmi les physica, au neutre pluriel, c' est-à-dire les ouvrages de philosophie naturelle; lesquels se rangent avec la Métaphysique parmi les ouvrages de philosophie théorique; lesquels, avec les œuvres de philosophie pratique et poétique constituent l' ensemble du corpus aristotélicien; lequel, augmenté de l'œuvre de divers ouvrages d' autres philosophes, aristotéliciens comme Alexandre d'Aphrodise ou néoplatoniciens comme Porphyre, grecs comme ces derniers, arabes comme Averroès, latins comme Boèce, forme le corpus péripatéticien ${ }^{46}$.

On pourrait continuer: le corpus péripatéticien n'est au fond que la partie principale d'un corpus philosophique plus large, qui inclut en outre, par exemple, ce qu'on peut lire de Platon. Et ce corpus philosophique, à son tour, n'est qu'une partie d'un corpus scolaire et universitaire, qui englobe en outre un corpus théologique, juridique, médical... Bref, toute la culture savante médiévale est un corpus de corpus, une hiérarchie textuelle à multiples étages, une sorte de super-œuvre collective et ramifiée, à la manière de Wikipedia. La notion d' «œuvre» peut donc, selon les points de vue, s' appliquer à divers étages de l' arborescence et la notion de tradition exégétique, comme les œuvres sur lesquelles elle porte, peut aisément glisser d'un niveau à l' autre, en sorte qu' on ait un grand nombre de traditions exégétiques imbriquées les unes dans les autres: celle du De memoria et reminiscentia dans celle des parva naturalia, celle des parva naturalia dans les physica d'Aristote, et ainsi de suite ${ }^{47}$.

Cette flexibilité de la notion d'œuvre, à l'intérieur d'un savoir encyclopédique, structuré en disciplines gigognes, dont chacune correspond à une auctoritas distincte, influe fortement sur la manière de lire et de commenter chaque œuvre séparée. Soit par exemple la question de la paternité du Liber de causis.

44 Theobald 1990; McDonald 2007.

45 Sur la question d'un catalogue et d'une édition par Andronicos de Rhodes des œuvres d'Aristote, voir en dernier lieu Kotwick 2016, p. 15-19.

46 D' où la vogue des divisiones philosophiae comme celles éditées par Lafleur 1988.

47 Voir entre autres, Dahan 199oc. 
On peuts' étonner que les commentateurs aient si longtemps persisté à prendre ce texte pour une œuvre d'Aristote, après que Thomas d'Aquin eut administré la preuve qu'il se rattache à l'école de Proclus ${ }^{48}$. Mis à part le conservatisme des universitaires médiévaux, cette constance dans l' attribution au Stagirite s' explique beaucoup mieux si on la rattache à la question des corpus.

Quand le Liber de causis apparaît dans les universités, dès le milieu du XIII ${ }^{\mathrm{e}}$ siècle, Aristote est le Philosophe par excellence, un auteur dont l'œuvre forme la base de l'enseignement d'à peu près toutes les parties de la philosophie. En sens inverse, Proclus sort à peine de l'ombre grâce aux traductions de Guillaume de Moerbeke ${ }^{49}$. Soit donc on tient le Liber de causis comme une œuvre sans père connu; ou, ce qui revient presque au même, comme un œuvre qui n'a pas pour père un auteur reconnu, faisant autorité en philosophie: il n' est donc pas utile de le commenter. Soit au contraire on se débrouille pour rattacher l'œuvre, de près ou de loin, au patronage d'Aristote: son commentaire devient alors souhaitable. La question de l'attribution du Liber de causis n' est pas tant, comme pour nous, une question d'histoire - histoire littéraire, histoire de la philosophie - qu' une question de corpus. Le contenu des manuscrits l'atteste, les commentaires du Liber de causis sont moins une tradition exégétique propre qu'une partie de la tradition exégétique du corpus aristotélicien. Il est donc normal que, parmi ceux qui choisissent de commenter le Liber de causis, l' attribution à Aristote soit fréquente. Pour ramener cette attribution à de justes proportions, il faudrait prendre en compte, non seulement les exégètes qui commentent cet ouvrage sans l' attribuer au Stagirite, mais encore ceux qui commentent Aristote sans commenter cet ouvrage, car ce choix négatif est en soi une réponse à la question de l' authenticité.

En somme, l' engouement pour le Liber de causis et les principales difficultés d' interprétation qu' il a suscitées s' expliquent largement par une logique de corpus: il remplissait si heureusement une lacune dans le panorama des études scolaires, l'absence d'une véritable théologie d'Aristote, qu'il devait être d'Aristote, d' une manière ou d' une autre. La tradition exégétique du corpus aristotélicien explique, pour une large part, la tradition exégétique du Liber de causis.

48 Voir D'Ancona 1992; Ead. 2002.

49 On trouvera un inventaire des éditions de Guillaume de Moerbeke comme traducteur de Proclus à l' adresse: https:/hiw.kuleuven.be/dwmc/ancientphilosophy/proclus/ proclused.html. 
Il est temps de conclure. Grâce à Dragos Calma et à son équipe, un objet neuf, considérable, est apparu dans le champ de l'histoire de la philosophie au Moyen Âge: au lieu d'une demi-douzaine de commentaires isolés sur le Liber de causis, c' est tout un filon exégétique qui vient au jour, bien plus ample, bien plus durable, bien plus complexe et enchevêtré qu' on ne le pensait. À objet nouveau, méthodes nouvelles: c'est à une réflexion sur les méthodes que j' ai voulu apporter ma contribution. Élaborée à partir d'un petit nombre de textes édités, enrichie par la comparaison avec d'autres traditions exégétiques, la réflexion demande à être discutée, affinée, corrigée, poursuivie au fil des nombreuses découvertes à venir. J' ai couru la course que je pouvais courir; maintenant je cède volontiers à d' autres la parole et le relais. 


\section{Appendice 1: Un exemple de tradition exégétique : le corpus dionysien en Occident du VI ${ }^{\mathrm{e}}$ au début du XIII ${ }^{\mathrm{e}}$ siècle}

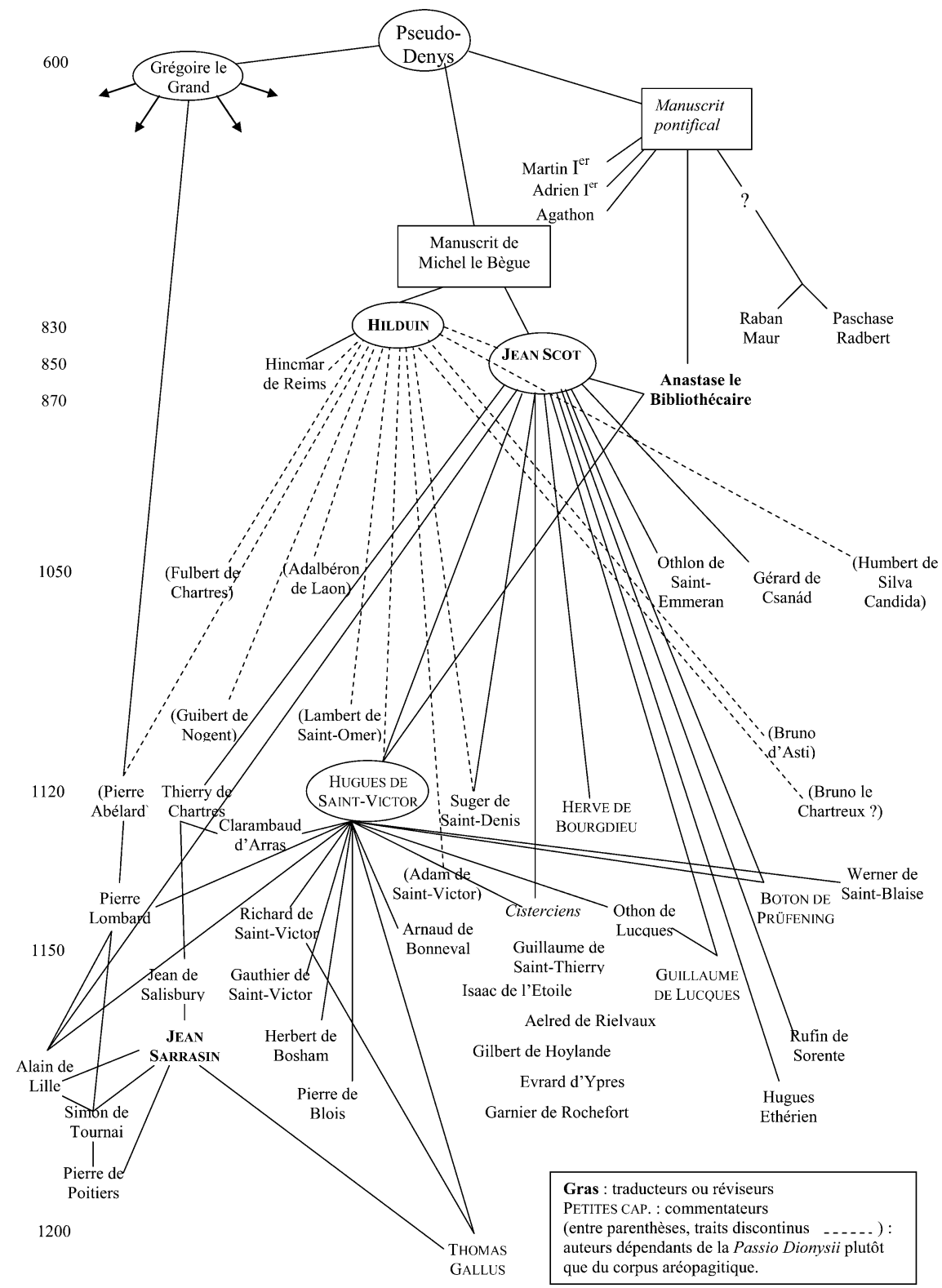




\section{Appendice 2: Édition provisoire du commentaire par Crisostomo Javelli de la première proposition du Liber de causis d' après l'édition de Venise 1531 , f. $2^{r}-43^{v}$}

Nous avons conservé l'orthographe de l' ouvrage imprimé. En revanche la ponctuation, qui sert à rendre le texte intelligible, est nôtre. Lorsque le texte nous a paru devoir être corrigé, nous l'avons fait dans le texte, en signalant la leçon d'origine en note.

Epithoma reverendi patris fratris Crisostomi Javelli Canapitii, ordinis predicatorum, sacre theologie professoris celeberrimi super propositiones

Libri de Causis et unicuique propositioni apposito commento, ac etiam examina earumdem.

\section{Propositio prima.}

$8^{\mathrm{r}} \quad$ Propositiones contente in Libro de causis, qui a pluribus asseritur compositus, a philosopho Arabe, sive ab Algazelle, sive ab Alpharabio, sive ab Avenpace, multas continet ambiguitates; adeoque difficile est scire in quo sensu acceptande vel reprobande sunt plures ex eis. Propterea decrevi singulas examinare secundum quadruplicem viam, scilicet secundum mentem authoris et Proculi platonici, qui et librum consimilem composuit, quem nominavit Elementationes theoricas, et Aristotelis et Theologi, ut manifestum sit secundum quam viam unaqueque vera vel non vera sit, et in quo sensu. Sed antequam descendam ad examen, prepono duo. Primo, quod in

Libro de causis cuilibet propositioni apponitur proprium commentum, quod iudicio meo editum est ab eodem authore quicumque fuerit. Nam quando commentator alius est ab authore, aliquando nominat ipsum, referens se ad eum; nominat, dico, aut in individuo, si habetur nomen eius, aut saltem in hoc titulo: «author huius libri etc.». In hoc autem commento, ut patet legenti, nunquam est relatio ad authorem, immo loquitur ut author exponens dictum suum. Quapropter in examine non distinguemus inter propositionem et commentum, sed existimabimus quod dicitur in commento dictum esse ab authore, et esse eiusdem veritatis vel falsitatis vel ambiguitatis cum propositione commentata. Secundo, quod non intendo prosequi nec exponere singula verba, sed per modum resolutorium complecti paucis sen-

$8^{\mathrm{rb}} \quad$ tentiam authoris, et deinde comparare eam doctri|ne Proculi et Aristotelis et Theologi. 


\section{Propositio PRima}

\section{OMNIS CAUSA PRIMARIA PLUS EST INFLUENS etc.}

In hac prima propositione intendit manifestare ordinem et differentiam inter causas primas et secundas, et intendit tres conclusiones.

Prima est: omnis causa primaria plus est influens super causatum quam causa universalis secunda.

Secunda est: cum removet causa universalis secunda virtutem a re, causa prima universalis non removet virtutem ab ea.

Tertia est: causa universalis prima agit in causatum cause secunde antequam agat in ipsum causa secunda que sequitur ipsam.

Pars prima secundum mentem authoris probatur quidem exemplo deducto ab esse et vivo et homine; quod, ut clarum, relinquimus. Probatur autem ab eodem ratione que consistit in hoc. Causa prima adiuvat causam secundam in actione sua, et non econverso. Item quamcumque actionem habet causa super effectum suum, habet et causa prima, quoniam in causis per se subordinatis, de quibus hic intendimus, causa media non causat nisi causante causa prima. Est enim causa media ut movens motum. Ergo causa prima plus influit, nam causat a se; secunda autem ut recipit a prima.

Secundum mentem Proculi, qui in libro suo de Elementatio theorica talem propositionem format: "Omne productum a causis secundis a prioribus et universalioribus producitur eminentius ${ }^{50}$ ». Sic probatur. Omnis causa secunda est effectus cause prime. Ergo habet substantiam et virtutem activam a causa prima. Ergo virtus agendi maior et nobilior est in prima causa quam in secunda. Sed virtus maior magis et eminentius producit effectum. Unumquodque enim agens agit secundum modum virtutis sue. Cum igitur idem sit effectus qui producitur a causa prima et a causis secundis subordinatis, magis et eminentius producitur a causa prima quam a secundis.

Secundum doctrinam Aristotelis, adverte quod, licet formaliter non habeamus hanc propositionem, tamen fundamentaliter haberi potest ex primo Posteriorum cum inquit: «Propter quod unumquodque et illud magis ${ }^{51}{ }_{\gg}$. Sed in proposito causa secunda agit propter primam, quia in virtute prime. Ergo prima magis agit in effectum quam secunda. Item in $12^{\circ}$ Methaphisice, tex. $34^{\circ}$ investigans agens perpetue generationis, determinat

28 adverte] conieci, auderte $e d$.

50 Cf. Proclus, Elementatio theologica, prop. 7.

51 Cf. Aristoteles, Analytica Posteriora, I, 2 (72a). 
quod, quoniam agens se habens alter et alter perpetuat generationem in virtute primi agentis, id est motoris primi celi semper eodem modo se habentis, illud primum agens dignius et eminentius se habet in perpetuando generationem ${ }^{52}$. Que quidem determinatio fundatur super hoc: primum agens, dans virtutem secundo, magis et dignius agit respectu eiusdem effectus ab utroque producti.

Secundum doctrinam Theologi, adverte quod, cum omnes cause secunde sint a prima create, comparantur ad ipsam sicut ministri ad regem et sicut organa ad principale agens; propterea movet et inclinat omnia in proprios fines, sed effectus productus a causa principali et ab organica magis et nobilius pendet a principali quam ab organica. Unde digestio magis est ab anima quam a calore naturali qui agit in virtute anime. Preterea, causa prima agit a se et independenter, cum non recipiat virtutem ab alio; causa autem secunda agit dependenter. Ergo dignius et magis influit in effectum quam causa secunda.

Hec igitur conclusio acceptanda est secundum omnem sanam doctrinam, quoniam est simpliciter vera.

Pars secunda ad mentem authoris sic probatur. Causa prima vehementius influit in effectum et in eo imprimit virtutem suam quam causa secunda, ex conclusione precedenti. Ergo virtus prime cause magis inheret effectui quam virtus cause secunde. Quod enim intensius imprimitur magis inheret, sicut calor intensus causatus in aqua magis inheret quam calor remissus. Sed quod magis inheret tardius removetur, ut patet de calore intenso. Ergo virtus prime cause tardius removetur ab effectu quam virtus cause secunde. Ex consequenti non est necesse, ad remotionem virtutis cause secunde ab effectu, removeri ab eodem virtutem prime cause.

Secundum mentem Proculi, qui in libro suo tenet impressionem prime cause ultimo recedere ab effectu ${ }^{53}$. Sic probatur. Causa perfectior extensius et intensius operatur. Patet de sole: qui, cum habeat lucem perfectissimam, $8^{\mathrm{vb}}$ illuminat intensius et extensius, quoniam, quantum $\mid$ sit ex se, illuminat omnia. Sed causa prima perfectior est causa secunda: dat enim sibi virtutem et non econverso. Ergo intensius et extensius operatur, quoniam operatur in omnibus, ut dicemus infra. Sed huiusmodi operans tardius recedit ab operato, ut patet de sole; cuius lux, cum sit intense et extense illuminans,

28 extensius] conieci, exteensius $e d$.

52 Cf. Aristoteles, Metaphysica, XII, 6 (1072a).

53 Cf. potius Thomas Aquinas, In Librum de causis, I, p. 5, l. 15; p. 7, l. 17-18; p. 8, l. 10-11. 
ultimo cessat illuminare. Nam extinctis sive remotis quibuscunque illuminantibus particularibus, ut igne, luna, astris, adhuc lux solaris illuminat. Ergo virtus prime cause impressa in effectu tardius removetur quam virtus cause secunde.

Secundum doctrinam Aristotelis, sic probatur. Causa prima comparatur ad secundam sicut causa prior ad posteriorem. Cum enim non sit processus in infinitum in causis per se subordinatis, ex $2^{\circ}$ Methaphisice, tex. $6^{\circ}$, necesse est omnem causam secundam habere rationem medii aut ultimi, ex consequenti esse posteriorem prima $^{54}$. Sed non est necesse ad remotionem posterioris removeri prius, quoniam non pendet a posteriori, et maxime in causis agentibus de quibus nunc loquimur, et verificatur in pluribus aliis. Unde Aristoteles, in $8^{\circ}$ Phisice, tex. $5^{\circ}$, probat motum localem esse primum, quoniam nullus alius motus remanet motu locali remoto, sed bene econverso ${ }^{55}$. Et in $2^{\circ}$ De anima, text. $31^{\circ}$, probat tactum esse primum sensum quoniam, remoto omni alio sensu, adhuc invenitur tactus. Unde, inquit, «Tactus quidem inest sine aliis. Multa enim animalium neque visum neque auditum habent neque odoratus sensum $^{56} »$. Cum igitur causa secunda posterior sit causa prima, non est necesse ad remotionem virtutis cause secunde, removeri virtutem cause prime.

Secundum doctrinam Theologi sic probatur. Causa prima proprie et principaliter est causa rei secundum esse et secundum produci. Causa autem secunda proprie est causa secundum produci, ut patet in omnibus agentibus particularibus artificiatis et naturalibus. Licet enim sol et homo generent hominem, tamen homo genitus conservatur a sole motu suo, et non ab homine generante; sed remota virtute cause in fieri, non est necesse removeri virtutem cause in esse et in fieri; aliter completa rei productione statim desineret esse. Ergo remo-|-ta virtute cause secunde, non est necesse removeri ab effectu virtutem cause prime.

Hec igitur conclusio, sicut et precedens, acceptanda est secundum omnem sanam doctrinam, quoniam est simpliciter vera.

Pars tertia patitur difficultatem propter ly 'antequam' sive 'prius'. Dupliciter enim potest exponi, scilicet 'prius in quo', et 'prius a quo'. Si 'prius in quo', conclusio erit falsa. Sensus enim erit: prima causa pro aliquo instanti vel tempore producit effectum cause secunde, pro quo nundum producitur a

54 Cf. Aristoteles, Metaphysica, XII, II, 2 (994a).

55 Cf. Aristoteles, Metaphysica, viII, cap. viI (206b).

56 Cf. Aristoteles, De anima, II, cap. II, par. 4 (413b); par. 9 (414a); cap. III, par. 2 (414b); cf. potius Thomas Aquinas, Commentaria in octo libros Physicorum, II, lec. 3, par. 16. 
causa secunda; et id non est verum, quoniam secunda causa non ageret in effectum suum si iam productus fuisset a prima, aliter productum produceretur, quod implicat. Si autem sumatur 'prius a quo', conclusio erit vera. Sensus enim est: prima causa agit a se in effectum cause secunde. Sed secunda causa non agit a se. Exponatur ergo ly 'prius' vel 'antequam', non secundum durationem, sed secundum causalitatem. Et hec quidem videtur fuisse mens authoris. Nam sic eam probat: causa secunda non agit in causatum suum nisi quia prima causa influit virtutem suam super eam. Ergo causa prima facit ut effectus attingatur a causa secunda. Ergo prius attingitur a causa prima. Patet autem hanc probationem non valere, nisi exponendo ly 'prius' causaliter et originative.

Secundum mentem Proculi, qui in libro suo talem propositionem format: «Omnis causa ante causatum operatur ${ }^{57}$, aliter probanda est. Sed antequem probetur, adverte quod Proculus per ly ‘omnem causam' intendit causam que tantum est causa; et per ly 'causatum' intendit causam que est effectus et causa: qualis est causa secunda, que est effectus prime et causa alterius, ut in hiis corporalibus: homo generans est effectus, et est causa hominis geniti. Per 'causam' igitur Proculus intendit causam primam, et per 'causatum' intendit causam secundam. Sic autem probat eam : causa prima est magis causa quam causa secunda. Ergo est perfectioris virtutis. Sed perfectior virtus ad plura se extendit, ut patet de luce solis, et prius advenit ei in quod agit. Ergo causa prima operatur antequam operetur causa secunda. Manifestum est autem quod hec probatio non concludit nisi de prioritate causalitatis, quoniam de prioritate durationis hec consequentia non valet:

$9^{\mathrm{rb}} \quad$ causa prima est perfe-|ctioris virtutis, ergo prius duratione operatur in effectum cause secunde quam causa secunda; aliter idem effectus iam productus adhuc produceretur, vel causa secunda superflueret.

Secundum doctrinam, sic probatur. Qualis est ordo inter eas subordinatas, talis est inter earum effectus, quoniam unicuique correspondet proprius effectus. Unde in $2^{\circ}$ Phisice tex. $35^{\circ}$ docuit reducere effectus in causas suas correspondentes, ut effectum particularem in causam particularem et effectum universalem in causam [in] universalem et priorem in causam priorem et posteriorem in posteriorem ${ }^{58}$. Sed inter causam primam et causam secundam servatur ordo prioris et posterioris. Nam cum sol et homo generant hominem, sol est causa prior, quoniam universale prius est, et homo

29 proprius] conieci, proprios ed.

57 Cf. Proclus, Elementatio theologica, prop. 57.

$5^{8}$ Cf. potius Thomas Aquinas, Commentaria in octo libros Physicorum II, cap. III, lec. 6, par. 11, p. $74,2^{\mathrm{e}}$ col. 
est causa posterior. Ergo effectus cause prime est prior et effectus cause secunde est posterior, et posito quod idem effectus sit ab utraque, comparatus cause prime fiet prius, et comparatus cause secunde fiet posterius. Quod quidem 'prius' intelligendum est, ut diximus, quo ad causalitatem. Causa enim secunda non agit nisi presuposita receptione virtutis a causa prima. Ex consequenti quo ad causalitatem posterius agit, licet pro eodem instanti simul agant. Causa autem prima agit non supposita receptione virtutis ab alio priori, ergo etc.

Secundum doctrinam Theologi sic probatur. Causalitas supponit esse, quoniam actio est existentis, cum unumquodquod agat inquantum est actu. Ergo sicut se habet esse cause secunde ad esse cause prime, ita causalitas cause secunde ad causalitatem prime. Sed esse cause secunde posterius est esse cause prime, quoniam est effectus cause prime, ut dicemus infra. Ergo causalitas cause secunde super effectum suum posterior est causalitate cause prime. Et adverte hanc deductionem concludere de prioritate causalitatis et non durationis, quoniam non repugnat dari effectum coevum cause agenti et producenti sine motu; et ideo non est necesse quod esse et causalitas cause secunde sint posteriora duratione esse et causalitate cause prime.

Predicta igitur tertia conclusio supra, quo ad prioritatem causalitatis concedenda est secundum omnem sanam | doctrinam, quoniam est simpliciter vera sicut et precedentes. Hec dicta sint super primam propositionis authoris.

1 effectus] conieci, effectos ed. 


\section{Bibliographie}

\section{Manuscrits}

Bologna, Biblioteca Universitaria, 1180 (2344)

London, Wellcome Historical Medical Library, 3

Paris, Bibliothèque nationale de France, lat. 17232

Vaticano (Città del), Biblioteca Apostolica Vaticana, Vat. lat. 5988

\section{Sources primaires}

Chrysostomus Javellus Canapicius, Epithoma Reverendi Patris Fratris Crisostomi Javelli Canapitii: Ordinis Predicatorum: Sacre Theologie Professoris Celeberrimi: super Propositiones Libri de Causis: et unicuique Propositioni apposito Commento: ac etiam Examina earumdem, Venezia, in aed. Francisci Bindoni 1531.

Thomas d'Aquin, Super Librum de causis expositio, éd. H.D. Saffrey, Fribourg / Louvain, Société philosophique / E. Nauwelaerts, 1954 [2 $2^{\mathrm{e}}$ Paris, Vrin, 2002]

Thomas d'Aquin, Commentaria in octo libros Physicorum, éd. Commissio Leonina, Opera Omnia, t. II, Roma, Ex Typographia Polyglotta, 1884.

\section{Sources secondaires}

Atucha, I., Calma, D., König-Pralong, C., Zavattero, I. (éds) (2011) Mots médiévaux offerts à Ruedi Imbach, Turnhout / Porto, Brepols, 2011.

Bastiaensen, A. (1990), «La perdrix animal méchant figure du diable: Augustin héritier d' une tradition exégétique», dans Augustiniana, t. 40, p. 193-217.

Bataillon, L.J., Dahan, G., Gy, P.-M. (éds) (2004), Hugues de Saint-Cher (†1263), bibliste et théologien. Études réunies, Turnhout, Brepols.

Bianchi, L., Randi, E. (1990), Le verità dissonanti. Aristotele alla fine del medioevo, Roma / Bari, Laterza.

Bianchi, L. (2003), Studi sull'aristotelismo del Rinascimento, Padova, Il Poligrafo, 2003.

Bianchi, L. (éd.) (2011), Christian Readings of Aristotle from the Middle Ages to the Renaissance, Turnhout, Brepols.

Biard, J. (1986), «La logique de l' infini chez Jean Mair», dans Les Études philosophiques, p. 329-348.

Boulègue, L. (éd.) (2014), Commenter et philosopher à la Renaissance. Tradition universitaire, tradition humaniste, Villeneuve d'Ascq, Presses Universitaires du Septentrion.

Boulnois, O., Schmutz, J., Solère, J.-L. (éds) (2002), Le contemplateur et les idées. Modèles de la science divine, du néoplatonisme au XVIII e siècle, Paris, Vrin.

Boureau, A., Piron, S. (éds) (1999), Pierre de Jean Olivi (1248-1298). Pensée scolastique, dissidence spirituelle et société. Actes du colloque de Narbonne (mars 1998), Paris, Vrin, 1999 . 
Brînzei, M. (2011), «Plagium», dans Atucha, Calma, König-Pralong, Zavattero (2011), p. $559-568$.

Calma, D. (éd.) (2016), Neoplatonism in the Middle Ages. New Commentaries on Liber de causis and Elementatio Theologica, 2 vol., Turnhout, Brepols.

Calma, D. (2016a), «The Exegetical Tradition of the Medieval Neoplatonism: Considerations on a Recently Discovered Corpus of Texts », dans Calma 2016-1, p. 11-52.

Cantelli, S. (1990), Angelomo e la scuola esegetica di Luxeuil, Spoleto, Fondazione CISAM, t. 1.

Cheng, A. (1985) Étude sur le confucianisme Han: l'élaboration d'une tradition exégétique sur les classiques, Paris, Institut des Hautes Études Chinoises / Collège de France.

Chenu, M.D. (1955), «Involucrum: le mythe selon les théologiens médiévaux», dans Archives d' histoire doctrinale et littéraire du Moyen Âge 3०, p. 75-79.

Cocteau, J. (1953), Journal d'un Inconnu, Paris, Grasset [réimp. Paris, Grasset, 2003].

Cocteau, J. (1927), Opéra; CEuvres poétiques, Paris, Stock [rééd. Paris, Stock, 1967].

Contamine, G. (éd.) (1989), Traduction et traducteurs au Moyen Âge. Actes du colloque international du CNRs organisé à Paris, Institut de recherche et d'histoire des textes les 26-28 mai 1986, Paris, Éditions du CNRs.

Dahan, G. (1990), «La classification du savoir aux XII ${ }^{\mathrm{e}}$ et XIII ${ }^{\mathrm{e}}$ siècles», dans L'enseignement philosophique, t. 40, p. $5^{-27}$.

Dahan, G. (1999a), « Olivi utilisateur de la Catena aurea de Thomas d' Aquin», dans Boureau, Piron (1999), p. 113-114.

Dahan, G. (1999b), L'exégèse chrétienne de la Bible en Occident médiéval, XII ${ }^{e}-X I V^{e}$ siècle, Paris, Éditions du Cerf, 1999.

Daley, B.E., Kolbet, P.R. (éds) (2014), The Harp of Prophecy: Early Christian Interpretation of the Psalms, South Bend, University of Notre Dame Press.

D’Ancona, C. (1992), «Saint Thomas lecteur du Liber de causis», dans Revue thomiste 92, p. 785-817, rep. in Ead., Recherches sur le Liber de causis, Paris, Vrin, 1995.

D’Ancona, C. (2000), «Syrianus dans la tradition exégétique de la Métaphysique d' Aristote», dans Goulet-Cazé 200o, p. 311-327.

D’Ancona, C. (2002), «Proclus, Denys, le Liber de Causis et la science divine», dans Boulnois, Schmutz, Solère 2002, p. 19-44.

Dondaine, H.F. (1953), Le Corpus dionysien de l'université de Paris au XIII e siècle, Roma, Edizioni di Storia e Letteratura.

Ernout, A., Meillet, A. (2001), Dictionnaire étymologique de la langue latine. Histoire des mots, Paris, Klincksieck.

Frank, G., Speer, A. (éds) (2007), Der Aristotelismus in der frühen Neuzeit, Kontinuität oder Wiederaneignung?, Wiesbaden, Harrassowitz.

Galle, G. (2008), «Edition and Discussion of the Oxford Gloss on De sensu 1», dans Archives d'Histoire Doctrinale et Littéraire du Moyen Âge, t. 75, p. 197-281. 
Gastaldelli, F. (1983), Wilhelmus Lucensis, Comentum in tertiam ierarchiam Dionisii que est De divinis nominibus. Introduzione e testo critico, Firenze, Olschki.

Goulet-Cazé, M.-O. (éd.) (2000), Le commentaire entre tradition et innovation. Actes du colloque international de l'Institut des traditions textuelles (Paris et Villejuif, 22-25 septembre 1999), Paris, Vrin.

Jeauneau, É. (1973), «L'usage de la notion d'integumentum à travers les gloses de Guillaume de Conches», dans Id., Lectio philosophorum. Recherches sur l'école de Chartres, Amsterdam, A.M. Hakkert, p. 127-192.

Kotwick, M.E. (2016), Alexander of Aphrodisias and the Text of Aristotle's Metaphysics, Berkeley, California Classical Studies.

Kristeller, P.O. (1960-), Catalogus Translationum et Commentariorum. Medieval and Renaissance Latin Translations and Commentaries, Washington, Catholic University of America Press.

Lafleur, C. (1988), Quatre introductions à la philosophie au XIII ${ }^{e}$ siècle. Textes critiques et étude historique, Montréal / Paris, Institut d' études médiévales / Vrin.

Lobrichon, G. (1990), «La bible d' un maître dominicain de Paris », dans Martin, Vezin 1990, p. 181-185.

Martin, H.-J., Vezin, J. (199o), Mise en page et mise en texte du livre manuscrit, Paris, Promodis.

McDonald, L.M. (2007), The Biblical Canon: its Origin, Transmission, and Authority, Peabody, Hendrickson Publishers.

Moulin, I. (éd.) (à paraître), Commenter au Moyen Âge. Colloque international de l'Institut d'études médiévales (IEM) de l'Institut catholique de Paris, en collaboration avec le Laboratoire d'études sur les monothéismes (LEM), 8-9 décembre 2014.

Nemeth, C. (2010), «The Victorines and the Areopagite», dans Poirel 2010, p. 333-383.

O'Keefe, J.J. (2014), «Theodoret's unique Contribution to the Antiochene Exegetical Tradition: Questioning Traditional Scholarly Categories», dans Daley, Kolbet 2014, p. 191-203.

Parisse, M., Goullet, M. (éds) (2001), Les historiens et le latin médiéval. Colloque tenu à la Sorbonne les 9, 10 et 11 septembre 1999, Paris, Publications de la Sorbonne.

Poirel, D. (1999), «Le "chant dionysien", du IX ${ }^{\mathrm{e}}$ au XII ${ }^{\mathrm{e}}$ s.», dans Pariss, Goullet 2001, p. $151-176$.

Poirel, D. (éd.) (2010), L'école de Saint-Victor. Influence et rayonnement du Moyen Âge à la Renaissance. Colloque international du c.N.R.s. pour le neuvième centenaire de la fondation (1108-2008), Turnhout, Brepols.

Poirel, D. (2013), Des symboles et des anges. Hugues de Saint-Victor et le réveil dionysien $d u X I I^{e} s .$, Turnhout, Brepols.

Rorem, P. (2009), Hugh of Saint Victor, Oxford, Oxford University Press.

Roques, R. (1962), «Connaissance de Dieu et théologie symbolique d'après l'In Hierarchiam coelestem sancti Dionysii de Hugues de Saint-Victor», dans Id., Structures 
théologiques de la Gnose à Richard de Saint-Victor. Essais et analyses critiques, Paris, Presses universitaires de France, p. 294-364.

Schmutz, J. (2001), «Bulletin de scolastique moderne», dans Revue thomiste, t. 100, p. 27-341.

Sirat, C., Geoffroy, M. (2005), L'original arabe du Grand Commentaire d'Averroès au De anima d'Aristote. Prémices de l'édition, Paris, Vrin.

Slotemaker, J., Witt, J. (éds) (2015), A Companion to the Theology ofJohn Mair, 2015, Leiden, Brill.

Smalley, B. (1961), «Les commentaires bibliques de l'époque romane: glose ordinaire et gloses périmées », dans Cahiers de civilisation médiévale, t. 4, p. 15-22.

Smith, L. (2009), The Glossa ordinaria: the Making of a Medieval Bible Commentary, Leiden, Brill.

Stock, B. (1972), Myth and Science in the Twelfth Century. A Study of Bernard Silvester, Princeton, Princeton University Press.

Tavuzzi, M. (1990) «Chrysostomus Javelli op (ca. 1470-1538). A Biobliographical Essay», dans Angelicum, t. 67, 1990, p. 347-378

Tavuzzi, M. (1991), «Chrysostomus Javelli op (ca.1470-1538). A Biobliographical Essay», dans Angelicum, t. 68, 1991, p. 109-121.

Tavuzzi, M. (2007), Renaissance Inquisitors:Dominican Inquisitors and Inquisitorial Districts in Northern Italy, 1474-1527, Leiden, Brill.

Theobald, C. (éd.) (1990), Le Canon des Écritures : études historiques, exégétiques et systématiques, Paris, Éditions du Cerf.

Thijssen, J.M.M.H., Braakhuis, H.A.G. (éds) (1999), The Commentary Tradition on Aristotle's De generatione et corruptione. Ancient, Medieval and Early Modern, Turnhout, Brepols.

von Wille, D. (2004), «Javelli, Giovanni Crisostomo», dans Dizionario Biografico degli Italiani, t. 62.

Weijers, O., Holtz, L. (éds) (1997), L'enseignement des disciplines à la Faculté des arts (Paris et Oxford, $X I I I^{e}-X V^{e}$ siècles), Turnhout, Brepols.

Weijers, O. (2011), Études sur la Faculté des arts dans les universités médiévales. Recueil d'articles, Turnhout, Brepols.

Weijers, O. (1996) Le maniement du savoir. Pratiques intellectuelles à l'époque des premières universités (XIII ${ }^{e}-X I V^{e}$ siècles), Turnhout, Brepols.

Zimmermann, M. (éd.) (2001), Auctor et auctoritas. Invention et conformisme dans l'écriture médiévale, Actes du colloque tenu à l'Université de Versailles - SaintQuentin-en-Yvelines, 14-16 juin 1999, Paris, École des Chartes. 